\title{
Open lacrimal surgery: a comparison of admission outcome and complications after planned day case or inpatient management
}

\author{
Ivan T Hanna, Suzanne Powrie, Geoffrey E Rose
}

\begin{abstract}
Aims-To assess the outcome, in terms of completion of admission and complication rates, for two series of patients undergoing open lacrimal surgery, one group planned for a day case admission and the other planned for inpatient stay. Methods-A retrospective analysis of case notes was performed for 200 patients planned to have day case admission (for 209 open lacrimal operations) and the details compared with those for 200 inpatient admissions (for 228 lacrimal procedures) during the same period of study. The success rates for surgery were not examined.

Results-9\% of planned day cases required overnight admission, $5.5 \%$ for medical reasons and $3.5 \%$ for social or administrative reasons. There was a similar incidence of postoperative complications in planned day cases and inpatients, although $6 \%$ of day case patients developed postoperative cellulitis, compared with $1.3 \%$ of the inpatients $(p=0.01)$. Overall, the incidence of cellulitis was greater in cases complicated by intraoperative haemorrhage $(p<0.05)$ or the use of absorbable nasal packing $(p<0.0001)$. A similar number of patients in each group attended the accident and emergency department before the planned first postoperative clinic visit.

Conclusion-Patients undergoing open lacrimal surgery can be safely managed as day cases if carefully selected for suitability on social and medical criteria. The use of general anaesthesia is not, in itself, a contraindication to day case lacrimal surgery.

(Br f Ophthalmol 1998;82:392-396)
\end{abstract}

Following recommendations by the Audit Commission, ${ }^{1}$ increasing numbers of cataract and squint operations are performed as day case or ambulatory procedures. In contrast, few surgeons perform external dacryocystorhinostomy (DCR) as a day case procedure and, in any such published series, the surgery is usually performed under local anaesthesia or assisted local anaesthesia. ${ }^{23}$

In this paper we present a retrospective analysis of our experience with day case open lacrimal surgery performed, in most cases, with general anaesthesia. The success rate for the surgery is not examined, as this paper is specifically intended to address the outcome in terms of completion of admission episodes and the rate of postoperative complications.

Patients and methods

Patients undergoing open lacrimal surgery over a 25 month period to January 1995 were identified using the operating theatre records and the hospital computer administration system. Two hundred consecutive day case admissions and 200 inpatient admissions were selected for comparison. The inpatient group was chosen by selecting, for each day case operation, the next inpatient procedure on the same operating list or, if no inpatient DCR was available or the case had already been selected, the next unselected inpatient DCR was chosen. At Moorfields Eye Hospital, there are several lacrimal theatre lists each week, each being allocated to a particular grade of surgeon, and the method for choosing inpatient "controls" was such that, where possible, each day case and "control" was treated by the same grade of surgeon and anaesthetist.

The surgery was performed by one surgical team under the supervision of a single consultant, the trainees comprising two postaccreditation specialist fellows and three residents (senior registrar, registrar, and senior house officer). All surgery performed by residents was supervised by a fellow or the consultant.

A uniform technique for external DCR was used. A vertical incision was placed $1 \mathrm{~cm}$ anterior to the medial canthus, the skin undermined posteriorly, the pretarsal and preseptal orbicularis muscle fibres separated and the medial canthal tendon disinserted from the nasal periosteum. A large rhinostomy was fashioned (16-18 mm diameter) and a partial ethmoidectomy performed; all bone alongside the lacrimal sac and the upper end of the nasolacrimal duct being removed. Anterior and posterior flaps were fashioned from lacrimal sac and nasal mucosa and sutured with interrupted $6 / 0$ Vicryl (Ethicon Co). The medial canthal tendon was reattached to preseptal orbicularis and the skin closed with a continuous, horizontal mattress $6 / 0$ nylon suture. Silastic tubes were used if there was common canalicular surgery, inflamed lacrimal sac mucosa or difficulty fashioning the mucosal flaps. Patients with canalicular disease either underwent canaliculo-dacryocystorhinostomy with intubation or DCR with placement of a Lester Jones tube.

To assess the efficacy of planned day case surgery, we reviewed the number of patients successfully treated as day cases and, in those 
patients admitted overnight, the reason for admission was determined. For comparison, we assessed how many of the inpatient group required additional inpatient stay beyond their planned discharge date, which was normally the first postoperative day. Any operative or postoperative complications were recorded and, in particular, the details of those who attended the accident and emergency department before the planned first postoperative clinic visit (normally 1 week after surgery) or required readmission to hospital. Cellulitis was considered to be present where there were increasing symptoms and clinical signs of postoperative tissue inflammation, these signs being thought to require systemic antibiotic therapy.

$\chi^{2}$ analysis or Fisher's exact test was used to compare various factors between the groups.

ANAESTHETIC METHODS

For adults in whom general anaesthesia was planned, their suitability was assessed at the preoperative outpatient visit by appropriately trained nurses, using a proforma, and with access to anaesthetic advice if required; a history of postoperative vomiting was not considered a contraindication to day case admission. On the day of surgery, the patient was assessed by the anaesthetist, with particular attention to any history of asthma or chronic obstructive airways disease (COAD). An oral premedication of an antiemetic, such as metoclopramide, was prescribed where there was a history of postoperative nausea or vomiting, but only rarely was a sedative given, as this tends to delay postoperative mobilisation. The necessity for early mobilisation after day case surgery precludes the use of long acting anaesthetic drugs, respiratory depressants, or the induction of intraoperative hypotension with ganglion blockade or large doses of $\beta$ adrenergic antagonists.

A useful practical management for both day cases and inpatients, giving relatively rapidly reversible anaesthesia and hypotension, was provided by induction with propofol and muscle relaxation with vecuronium bromide. Endotracheal intubation and a pharyngeal pack were generally placed after endotracheal intubation. Good principles of anaesthesia for head and neck surgery were followed-that is, a quiet induction and adequate time allowed for complete muscle relaxation before intubation of the trachea, thereby avoiding coughing and the consequent rise in venous pressure. Anaesthesia was maintained with isoflurane (up to $2.5 \%$ ) in nitrous oxide and oxygen. Rarely, a short acting opiate, such as alfentanil, was required as, for example, when there was a tachycardia in the presence of an adequate, but low, blood pressure. Mild hypotension was induced in suitable patients, where necessary, by giving (up to $5 \mathrm{mg}$ ) metoprolol tartrate in 1 mg aliquots until the mean blood pressure was in the order of $65-80 \mathrm{~mm} \mathrm{Hg}$; metoprolol was avoided in patients with asthma, COAD, or treated cardiac failure, and was used with caution in the elderly and those with treated hypertension. The available ganglion blocking drugs, such as pentolinium tartrate, were too long acting to be of value in day case anaesthesia and older drugs, such as trimetaphan, produced too great a reflex tachycardia. The use of a glyceryl trinitrate infusion was assessed before this study, but was abandoned because it gave a too great, and also unpredictable, hypotension with poor operating conditions.

The patient was positioned on the operating table with a head up tilt and the hypotensive effect of blood pooling in the leg veins was induced both by utilising the vasodilatory property of isoflurane and by varying the tilt of the table. After preparation of the sterile field, three dressed orange sticks were dipped into a $0.1 \%$ adrenaline solution and positioned high in the nasal space, anterior to the middle turbinate on the side to be operated. Where there was a history of postoperative nausea or vomiting, $4 \mathrm{mg}$ intravenous ondansetron was given. Provided there were no contraindications, $10 \mathrm{mg}$ ketorolac trometamol was administered shortly before the end of surgery; this generally provided adequate postoperative pain relief and only rarely was further analgesia required.

Children were treated in a similar fashion to adults. Premedication was rarely required, except that a topical local anaesthetic cream (such as EMLA or Ametop) was applied, under occlusion, to the back of each hand. Most children were accompanied to the anaesthetic room by a parent, anaesthesia was induced intravenously, or by inhalation of sevoflurane, and then maintained by isoflurane. The pharynx was packed firmly, not only to reduce loss of gases from the (uncuffed) endotracheal circuit, but also to reduce the exposure of staff, particularly surgical, to the anaesthetic mixture while working close to the patient. Very young children were not given $\beta$ adrenergic antagonists or ketorolac; postoperative analgesia, using rectal diclofenac sodium, was given (with parental consent) before the child woke from anaesthesia. As in adults, good operating conditions were generally achieved by the use of a quiet induction, a head up positioning during surgery, and intranasal application of $0.1 \%$ adrenaline.

For local anaesthesia, $0.5 \%$ bupivacaine, prepared with added 1:80 000 adrenaline, was used to infiltrate anteriorly and posteriorly to the medial canthus and at the nasociliary nerve in the superonasal aspect of the orbit; since this study, commercially prepared bupivacaine $0.5 \%$ with 1:200 000 adrenaline has been used and found to be equally effective. After spraying the nasal space with lignocaine nasal spray, it was packed with approximately 1 metre of half inch ribbon gauze soaked in either $4 \%$ or $10 \%$ cocaine solution.

\section{Results}

\section{GROUP A}

Day case open lacrimal surgery

Of patients undergoing day case surgery, 177 underwent unilateral DCR on one occasion, seven had unilateral DCR on two separate occasions, and nine patients underwent bilateral DCR (200 admissions; 193 patients; 209 
Table 1 Type of surgery in each group of 200 patient admissions

\begin{tabular}{lll}
\hline Type of surgery & $\begin{array}{l}\text { Group A: day case, 209 } \\
\text { operations (200 } \\
\text { admissions) }\end{array}$ & $\begin{array}{l}\text { Group B: inpatient, } \\
\text { 228 operations (200 } \\
\text { admissions) }\end{array}$ \\
\hline Unilateral cases: & $\begin{array}{l}(191 \text { admissions) } \\
\text { DCR with/without tubes }\end{array}$ & $\begin{array}{l}\text { (172 admissions) } \\
\text { Canaliculo-DCR }\end{array}$ \\
DCR with Lester Jones tube & 363 & 2 \\
DCR with retrograde canaliculostomy & 5 & 5 \\
Redo DCR & 5 & 11 \\
Bilateral cases: & 15 & 39 \\
DCR with/without tubes & $(9$ admissions) & $(28$ admissions) \\
Canaliculo-DCR & 16 & 43 \\
DCR with retrograde canaliculostomy & 0 & 1 \\
Redo DCR & 1 & 8 \\
\end{tabular}

Table 2 Recorded operative and postoperative events in the day case and inpatient groups

\begin{tabular}{lll}
\hline Recorded adverse events & $\begin{array}{l}\text { Group A: day case } \\
(209 \text { operations })\end{array}$ & $\begin{array}{l}\text { Group B: inpatient } \\
(228 \text { operations })\end{array}$ \\
\hline Operative difficulties: & $(32 / 209$ operations $)$ & $(25 / 228$ operations $)$ \\
Creation of mucosal flaps & 14 & 14 \\
Troublesome haemorrhage & 15 & 9 \\
Skin tear from traction & 2 & 1 \\
False canalicular passage & 1 & 1 \\
Postoperative events: & $(51 / 209$ cases $)$ & $(56 / 228$ cases $)$ \\
Cellulitis & $12^{\star \star}$ & $3^{\star \star}$ \\
Intermittent epistaxis & 6 & 9 \\
Nasal ooze (day 1-4) & 4 & 1 \\
Silicone tube problems & 5 & 12 \\
Noticeable skin scar & 15 & 14 \\
Significant bruising & 3 & 6 \\
Miscellaneous details & 6 & 11 \\
\hline
\end{tabular}

$\star \star \mathrm{p}=0.01$ (Fisher's exact test).

Table 3 Reasons for 18 overnight admissions in the group of 200 admissions planned to be day case

\begin{tabular}{lll}
\hline Type of surgery & Recorded adverse event & $\begin{array}{l}\text { Proportion of the (18) } \\
\text { planned day cases } \\
\text { admitted overnight }\end{array}$ \\
\hline Bilateral surgery (9 admissions) & (None) & $2 / 18$ \\
Unilateral surgery (191 admissions) & $\begin{array}{l}\text { Medical events (9 admissions): } \\
\text { Postoperative nausea/vomiting }\end{array}$ & $4 / 18$ \\
& Difficult intubation & $1 / 18$ \\
& Postoperative hypotension & $1 / 18$ \\
& Postoperative bradycardia & $1 / 18$ \\
& Postoperative drowsiness & $1 / 18$ \\
& Continued postoperative & $1 / 18$ \\
& haemorrhage & \\
& Non-medical events (7 admissions): \\
& Patient preference & $4 / 18$ \\
& Lack of transport & $1 / 18$ \\
& Administrative problems & $2 / 18$
\end{tabular}

Table 4 Reasons for attendance at the accident and emergency department before the first planned postoperative clinic visit

\begin{tabular}{lll}
\hline $\begin{array}{l}\text { Reason for early postoperative } \\
\text { visit }\end{array}$ & $\begin{array}{l}\text { Group A: day case (4/209 } \\
\text { operations) }\end{array}$ & $\begin{array}{l}\text { Group B: inpatient (6/228 } \\
\text { operations) }\end{array}$ \\
\hline Epistaxis & 1 & 4 \\
$\begin{array}{l}\text { Cellulitis } \\
\text { Patient concern about tubes }\end{array}$ & 1 & - \\
"Vague feeling in head" & 1 & - \\
Blood stained tears & 1 & - \\
\hline
\end{tabular}

procedures). The average age of patients at surgery was 50.5 years (range 2.5-86 years; median 51 years).

Various surgeries were performed (Table 1). All bilateral cases were under general anaesthesia, three unilateral cases under local anaesthesia, and the remaining 188 under general anaesthesia. In 32/209 (16\%) operations, operative difficulty was recorded in the case notes (Table 2). The commonest problems encountered were in fashioning mucosal flaps or intraoperative haemorrhage. A postoperative event was recorded in 51/209 (24\%) cases, varying in severity from minor bruising to epistaxis (Table 2).

Of the 200 patient episodes planned to be day cases, 18 (9\%) resulted in the patient being admitted overnight; two of the nine having bilateral surgery $(22 \%)$ were admitted, compared with $16 / 191(8.4 \%)$ of patients with unilateral surgery (Table 3). The two bilateral cases had uncomplicated surgery and there was no definite reason for admission, apart from the fact that they were bilateral cases and anaesthetic time was therefore longer. With unilateral cases, nine patients $(4.7 \%)$ were kept overnight for medical reasons and seven (3.6\%) for social or personal reasons.

Four patients were admitted with nausea, vomiting, or abdominal pain, four for anaesthetic reasons (difficult intubation due to epiglottic mass, postoperative hypotension, postoperative bradycardia, or drowsiness) and one patient (who was taking aspirin preoperatively) was admitted because of mild continued postoperative haemorrhage (Table 3). Of the seven patients admitted for non-medical reasons, four were because of patient preference and one because of a lack of transport home. Two were kept overnight for surgery the following morning, because afternoon operating lists overran their allotted time.

Four patients $(2 \%)$, all of whom had undergone unilateral surgery, attended the accident and emergency department before their first booked clinic visit (Table 4). One patient attended with epistaxis that settled spontaneously, one with cellulitis, one with a "vague feeling in the head", and one with blood stained tears. None of these patients required readmission to hospital.

GROUP B

Inpatient open lacrimal surgery

Of patients undergoing inpatient surgery, 152 underwent unilateral surgery, 10 had unilateral surgery on two separate occasions, and 28 had bilateral surgery; this represented 200 admissions for 190 patients, undergoing a total of 228 open lacrimal surgeries. The average age was 56.1 years (range 2.8-92 years; median 64 years). All bilateral cases were under general anaesthesia, 13 unilateral cases under local and the other 159 under general anaesthesia (Table 1). Operative difficulties were recorded in $25 / 228(11 \%)$ procedures and in $56(25 \%)$ an abnormal postoperative event was recorded, the types of event being similar to those for day case patients (Table 2). One patient, who had undergone bilateral DCR under general anaesthesia, developed a cerebrovascular accident in the immediate postoperative period, necessitating transfer to another hospital for medical management

Patients with planned inpatient stay for lacrimal surgery were normally discharged on the first postoperative day, although four patients ( $2 \%$ of admissions) remained in hospital longer than expected; $3 / 172(1.7 \%)$ of unilateral operations and $1 / 28(3.6 \%)$ of bilateral operations. One patient was kept in hospital because of a grand mal seizure, one because of a cerebrovascular accident (bilateral 
surgery), one because of continued postoperative haemorrhage requiring nasal packing and one remained in hospital awaiting transport home.

Six patients (3\% admissions), all of whom had undergone unilateral surgery, attended the accident and emergency department before their planned first postoperative clinic visit (Table 4). Four patients attended with epistaxis, of which three required admission and one settled spontaneously, and two patients attended with problems due to silicone tubes-one with the tubes displaced at the medial canthus and the other patient because he could not see the tubes in the nose.

\section{Discussion}

Although day surgery is gaining popularity for cataract and squint operations, few surgeons perform open lacrimal surgery as a day case or ambulatory procedure and, where day case lacrimal surgery is practised, it is most frequently performed under enhanced local anaesthesia. ${ }^{34}$ Day case open lacrimal surgery was introduced to Moorfields Eye Hospital in 1992 and in this paper we have analysed a large number of such operations, the majority performed under general anaesthesia, comparing them with our experience of a similar number of inpatient operations. Although a slight bias to older patients in the inpatient group, the two groups were similar with respect to the range of ages, but there are significantly more bilateral cases or complex procedures in the inpatient group (Table 1). Primary DCR represented $179 / 209(85 \%)$ of planned day case operations, compared with 158/228 $(69 \%)$ of inpatient procedures $(\mathrm{p}<0.01)$; likewise $9 / 200(4.5 \%)$ of planned day case admissions were for bilateral surgery, in contrast with $43 / 200(21.5 \%)$ of inpatient episodes $(\mathrm{p}<0.01)$.

Since the inpatient group (B) contained a greater number of more complex cases, one might expect a greater number of operative or postoperative difficulties in this group. The incidence of operative difficulty was, however, slightly lower in the inpatient group $(11 \%)$, compared with the day case group (16\%), and the incidence of recorded postoperative events was similar ( $24 \%$ and $25 \%$, respectively).

In this study, $9 \%$ of patients attending for planned day case surgery were admitted overnight $(5.5 \%$ for medical reasons and 3.5\% for social or personal reasons), this admission rate being similar to that reported for nonophthalmic day surgery. ${ }^{4}$ The commonest cause of admission was nausea and vomiting, or anaesthetic related problems (eight cases; $4 \%$ ) and only one patient required admission for a surgical reason (continued postoperative haemorrhage). These figures compare favourably with those of Dresner and colleagues, ${ }^{2}$ who report that $9 / 105(8.6 \%)$ patients undergoing outpatient DCRs required immediate admission to hospital- $5.7 \%$ for medical reasons (three for nausea, three for epistaxis) and $2.8 \%$ for social reasons. Our study differs markedly from that of Dresner, with $98.5 \%$ of our day case admissions having surgery under general anaesthesia, in contrast with $27 \%$ of Dresner's series ${ }^{2}$; allowing for this difference, the incidence of anaesthetic related admissions is very similar ( $4 \%$ and $2.8 \%$, respectively). Benger $^{3}$ reported the results of 90 outpatient DCRs, seven $(8 \%)$ of which were under general anaesthesia; only one patient required immediate overnight admission, with urinary retention after general anaesthesia.

Just as the recorded postoperative events were similar in the day case and inpatient groups, the number of patients attending the accident and emergency department before their planned first clinic visit is similar in the two groups. The number of patients attending with epistaxis was, however, greater in the inpatient groups, although this might be attributed to a higher proportion of more complex procedures. There were four cases of delayed epistaxis in the inpatient group: two cases were following primary DCR and two cases following more complex surgery. One of the complex cases developed epistaxis after canaliculoDCR, having previously had radiotherapy for malignancy at the medial canthus-where one might reasonably expect delayed healing and an increased risk of secondary haemorrhage. The other complex inpatient case had epistaxis after redo DCR and it is of interest that, in the day case group (group A), the only case of secondary haemorrhage was also after redo surgery. Combining the data for inpatients and day cases, the incidence of delayed epistaxis was significantly greater after more complex open lacrimal surgery (redo DCR, canaliculoDCR, DCR with Jones tubes)—namely, 3/159 $(1.8 \%)$ of complex cases, compared with $2 / 278$ $(0.7 \%)$ of primary DCRs $(p=0.35)$. Twelve of the 209 planned day case DCRs (6\%) developed postoperative cellulitis, compared with $3 / 228(1.3 \%)$ inpatient cases $(\mathrm{p}=0.01)$. Patients were normally prescribed a broad spectrum systemic antibiotic after surgery, although two day case patients and one inpatient failed to receive adequate antibiotics and this might have contributed to their developing cellulitis. Intraoperative haemorrhage and the use of self dissolving nasal tamponade ("Spongostan"; Ferrosan) may also present another predisposition to postoperative infection.

The recorded incidence of intraoperative haemorrhage was greater with day cases $(15 / 209 ; 7 \%)$, compared with $9 / 228$ (4\%) inpatients $(\mathrm{p}=0.21)$ and in six day case patients, Spongostan was used to control haemorrhage, compared with no usage within the inpatient group. Combining data for day cases and inpatients, 3/23 (13\%) operations with recorded intraoperative haemorrhage developed postoperative infection, compared with $12 / 401 \quad(3 \%)$ without intraoperative haemorrhage $(\mathrm{p}<0.05)$. Of even greater significance, if soluble nasal tamponade was used, 6/11 (35\%) developed infection whereas only 9/420 (2\%) developed cellulitis where nasal tamponade was not used $(\mathrm{p}<0.0001)$. The threshold for actually recording intraoperative haemorrhage was probably lower for day case surgery and the apparently greater incidence of 
recorded haemorrhage may be an artefact. The use of soluble nasal tamponade appears, however, to be linked to the greater incidence of infection in the day case group; it being likely that a blood laden tampon at the rhinostomy site may provide an environment highly conducive to bacterial proliferation. Although antibiotic prophylaxis was prescribed, the drug may not have achieved adequate therapeutic levels within the blood clot and nasal tampon.

Day case open lacrimal surgery, whether a primary DCR or a complex procedure, would not appear to carry a greater risk of morbidity than inpatient care when cases are selected appropriately; indeed, we now perform the majority of such surgery, both for children and adults, as a day case. There are many factors to be considered in undertaking day case lacrimal surgery, including patient preference, social backup, and the surgeon's and anaesthetist's familiarity with the technique. Inpatient facilities are required for the few patients requiring admission, although non-medical admissions could be admitted to a hostel if available. In our series, a more careful preoperative assessment of a patient's social circumstances might have reduced unplanned admissions. Likewise, the patient requiring ambulance transport was probably unsuitable for day case surgery.

We believe that day case lacrimal surgery is a safe procedure, provided admission facilities are available, although careful preoperative assessment is required to minimise unnecessary medical or social admissions.

During the preparation of this work, Mr Ivan Hanna was supported by the Speed Pollock Memorial Research Trust and we are grateful to Dr L K Tu for his assistance with statistical analysis.

1 The Audit Commission. A short cut to better services-day surgery in England and Wales. London: HMSO, 1990:7.

2 Dresner SC, Klussman KG, Meyer DR Linberg JV. Outpatient dacryocystorhinostomy. Ophthalmic Surg 1991;22: 222-4.

3 Benger R. Day-surgery external dacryocystorhinostomy. Aust NZ F Ophthalmol 1992;30:243-5.

4 Williams $\mathrm{O}$, ed. New angles on day surgery. Anglia and Oxford Regional Health Authority and the NHS Executive, 1995:26. 